\title{
Disturbance of Cultured Rat Neuronal Network Activity Depends on Concentration and Ratio of Leucine and $\alpha$-Ketoisocaproate: Implication for Acute Encephalopathy of Maple Syrup Urine Disease
}

\author{
PHILIPP GÖRTZ, HUBERTUS KÖLLER, BERND SCHWAHN, UDO WENDEL, AND \\ MARIO SIEBLER \\ Departments of Neurology [P.G., H.K, M.S.] and Pediatrics [B.S., U.W.], Heinrich-Heine-University, \\ Düsseldorf, Germany
}

\begin{abstract}
Increased concentrations of leucine and its respective ketoacid $\alpha$-ketoisocaproate (KIC) in plasma and cerebrospinal fluid are related to acute and reversible encephalopathy in patients with maple syrup urine disease. We studied electrophysiological properties of primary dissociated rat neurons at increased extracellular concentrations of leucine and KIC (1-10 mM). Spontaneous neuronal network activity was reversibly reduced or blocked by leucine as well as by KIC in a dose-dependent manner. Simultaneous incubation with both substances led to a minor inhibition compared to the effect of each substance alone. Neuronal resting potential, voltage dependent $\mathrm{Na}^{+}\left(\mathrm{I}_{\mathrm{Na}}\right)$ and $\mathrm{K}^{+}$ $\left(\mathrm{I}_{\mathrm{K}}\right)$ currents, the GABA- and glycine-elicited membrane currents, and glutamate-induced intracellular $\mathrm{Ca}^{2+}$ increase of single neurons, however, were unaffected by both substances. We conclude that acute neuronal network dysfunction in maple syrup
\end{abstract}

\section{ABSTRACT}

urine disease is mainly based on an imbalance of the presynaptic glutamatergic/GABAergic neurotransmitter concentrations or their release. (Pediatr Res 53: 320-324, 2003)
Abbreviation
BCAA, branched-chain amino acid
BCKA, branched-chain keto acid
DF, depolarization frequency
$\mathbf{d F}$, difference of fluorescence ratios
DIV, days in vitro
$\mathbf{I}_{\mathbf{N a}}$, sodium membrane current
$\mathbf{I}_{\mathbf{K}}$, potassium membrane current
KIC, $\alpha$-ketoisocaproate
MSUD, maple syrup urine disease

Maple syrup urine disease (MSUD) is caused by an inherited deficiency of the branched-chain $\alpha$-keto acid dehydrogenase, resulting in a marked increase of the branched-chain amino acids (BCAAs), leucine, isoleucine and valine and their respective branched-chain $\alpha$-keto acids (BCKA) in body fluids. The disease is characterized by acute encephalopathy and chronic brain dysfunction. During episodes of acute encephalopathy with progressive neurologic signs - cerebral edema, seizures, and coma - the levels of BCAA and BCKA, particularly leucine and $\alpha$-ketoisocaproate (KIC), are greatly increased in blood, cerebrospinal fluid, and brain tissue. Immediate therapeutic reduction of toxic plasma

Received November 15, 2001; accepted July 22, 2002.

Correspondence: Mario Siebler, M.D., Department of Neurology, Heinrich-HeineUniversity Düsseldorf, P.O. Box 101007, 40001 Düsseldorf, Germany; e-mail: Siebler@uni-duesseldorf.de

Supported by grants of the Forschungskommission der Heinrich-Heine Universität Düsseldorf, the Ministerium des Landes Nordrhein Westfalen (Biochip Initiative) and the SHS-International (Germany).

DOI: 10.1203/01.PDR.0000047521.50656.16 levels relieves all signs of neurologic dysfunction within a few days. Protection of the patient against long-term myelin damage and mild retardation requires permanent treatment that aims to normalize plasma amino acid concentrations by adjusting dietary intake of BCAA (for review, see 1). The pathophysiological mechanisms underlying the acute dysfunction of the CNS are not clear, and various models are discussed: elevated levels of BCAA could decrease the transport of other large neutral amino acids across the blood-brain barrier (2), affect mitochondrial function (3), or induce neuronal apoptosis (4).

Leucine and/or KIC has been proposed as the cue substances in pathophysiology of neurologic dysfunction in MSUD (5). We studied the effects of increased extracellular leucine and KIC on spontaneous neuronal activity of cultured neuronal networks from embryonic rats. In a second step, we measured the influence of these substances on 
neuronal ion channels and the intracellular neuronal calcium response to glutamate.

\section{METHODS}

Cell culture. Neocortical neurons were prepared from embryonic Wistar rats at gestational day 15 and hippocampal neurons were prepared at gestational day 18 as described earlier (6). Briefly, embryos were removed, and their cortices or hippocampi were dissected. After trypsinization and trituration, the neuronal cell suspension was washed by filtration and centrifugation. Neurons were plated on glass coverslips, which were coated by PBS with poly-D-lysine $(0.1 \mathrm{mg} / \mathrm{mL})$ and laminin $(4 \mu \mathrm{g} / \mathrm{mL})$. The neurons were grown in serum-free supplemented $\mathrm{N} 2$ media in a humidified atmosphere $\left(5 \% \mathrm{CO}_{2} /\right.$ $95 \%$ atmosphere) at $37^{\circ} \mathrm{C}$, conditioned for at least $2 \mathrm{~d}$ by spatially separated cerebral astrocytes from newborn rats. The proportion of contaminating astrocytes was estimated to be $<5 \%$ in cultures up to 2 wk in vitro (6). Recordings were made between 8 and $12 \mathrm{~d}$ after preparation. All experimental procedures were approved by the local ethics committee.

Electrophysiological recordings. To investigate neuronal function during elevated levels of leucine and KIC in vitro, we used the method of the conventional whole-cell patch-clamp technique. All recordings were made at room temperature. Borosilicate pipettes (GC150-15; Clark Electromedical Instruments, Kent U.K.) were pulled to a tip diameter of $\sim 1-2 \mu \mathrm{m}$ corresponding to a resistance of 5-7 M $\Omega$. Membrane voltage and currents were amplified (EPC-7; List, Darmstadt, Germany), low-pass filtered at $2 \mathrm{kHz}$, digitized at $25 \mathrm{kHz}$ sampling rate, and stored on hard discs of a personal computer for further off-line analysis. The exchange of the extracellular solution in the bath chamber (volume of $1 \mathrm{~mL}$ ) was adjusted to a rate of $15-20 \mathrm{~mL} / \mathrm{min}$. The standard bath solution contained (in mmol/L) $150 \mathrm{NaCl}, 1 \mathrm{MgCl}_{2}$, $2 \mathrm{KCl}, 2.8 \mathrm{CaCl}_{2}, 10$ glucose, and 10 HEPES at $\mathrm{pH}$ 7.4. The pipette solution contained (in mmol/L) $150 \mathrm{KCl}, 4 \mathrm{MgCl}_{2}, 1$ $\mathrm{CaCl}_{2}, 10$ HEPES, and 10 EGTA at $\mathrm{pH}$ 7.4. The resting membrane potential was typical for primary dissociated neurons in cell cultures and ranged from -30 to $-60 \mathrm{mV}$.

Voltage-gated channels. Intracellular current pulses (20 ms) were applied in steps of $10 \mathrm{mV}$ from various holding potentials from -110 to $-20 \mathrm{mV}$. The peak values of the recorded inward and outward currents elicited from a holding potential of $-110 \mathrm{mV}$ were defined as the maximum of the voltagedependent $\mathrm{Na}^{+}$and $\mathrm{K}^{+}$currents (see Fig. 2 inset). Inward currents elicited at different holding potentials were fitted by a Boltzmann distribution, giving the value $\mathrm{V}_{1 / 2}$ of half maximal activation of voltage-dependent $\mathrm{Na}^{+}$channels. For the determination of the $\mathrm{Na}^{+}$-current inactivation, constant $\mathrm{V}_{1 / 2} \mathrm{~K}^{+}$ currents were blocked by replacing $\mathrm{KCl}$ in the patch pipette by $\mathrm{CsCl}$ and adding TEA $(10 \mathrm{mM})$ to the bath solution. After each run, tetrodotoxin (TTX, $1 \mu \mathrm{M}$ ) a specific blocker of the sodium channel, was added to the bath solution to ensure that the measured currents were sodium currents.

Resting membrane potential of neurons. Membrane potential was determined in the current-clamp mode after at least 5 min and up to $60 \mathrm{~min}$ of incubation in standard bath solution and compared with resting membrane potential of neurons

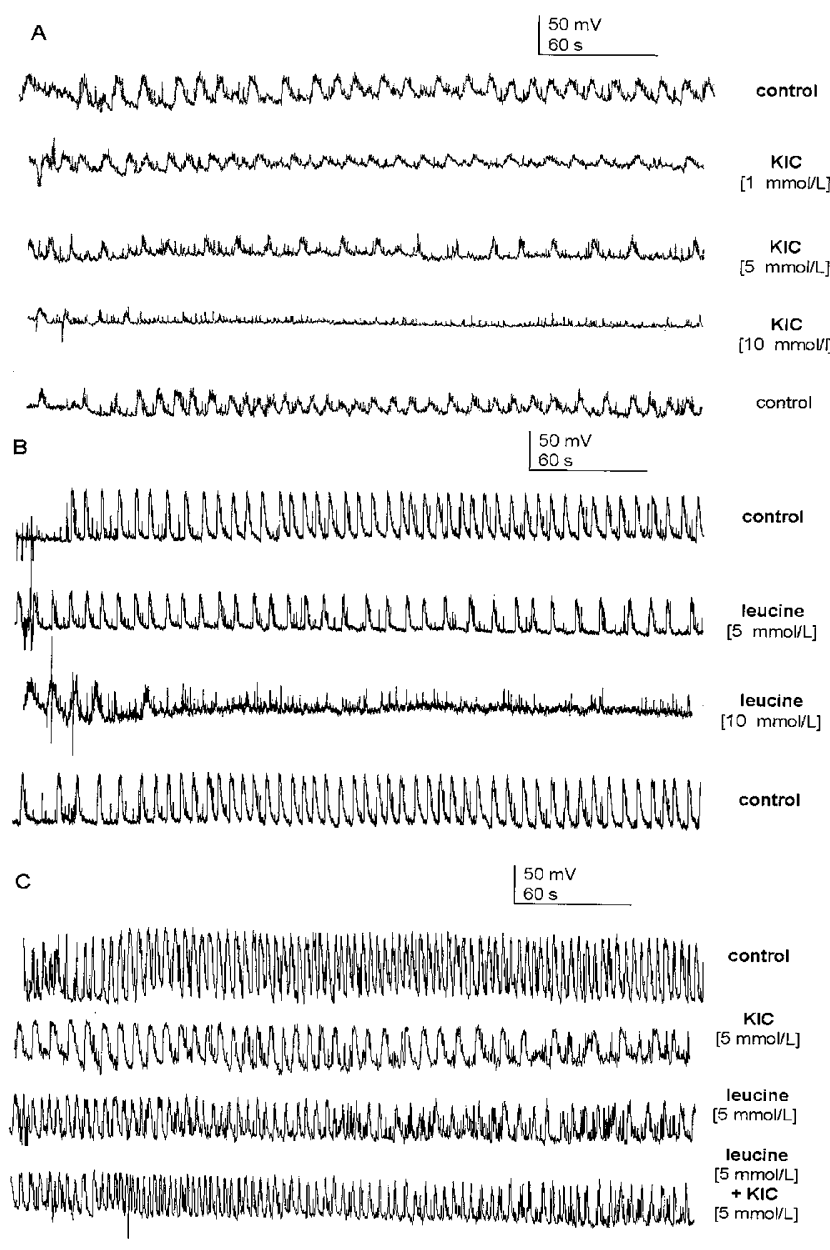

Figure 1. Leucine and KIC reversibly inhibited the DF of a neuronal network in a dose-dependent manner and partially compensated their effect. Whole-cell patch-clamp recordings in the current-clamp modus of an hippocampal neuronal network (DIV 12) in magnesium-free bath solution from 3 different neurons $(A-C)$. The traces display the periodic depolarization of membrane potential induced by action potential bursts of a hippocampal neuronal network. (A) Bath solution containing $\mathrm{KIC}(1 \mathrm{mM})$ reduced the DF from $6 \mathrm{D} / \mathrm{min}$ to $4 \mathrm{D} / \mathrm{min}, \mathrm{KIC}$ $(5 \mathrm{mM})$ reduced $\mathrm{DF}$ to $3 \mathrm{D} / \mathrm{min}$, and $\mathrm{KIC}(10 \mathrm{mM})$ abolished depolarization events. The effect was completely reversible (see lower trace after washing with control solution). (B) Similar effect was detected after incubation with leucine. Leucine $(5 \mathrm{mM})$ reduced DF from $10 \mathrm{D} / \mathrm{min}$ to $5 \mathrm{D} / \mathrm{min}$ and for leucine $(10 \mathrm{mM})$ depolarization vanished. $(C)$ Leucine and KIC partially compensated their effect on the inhibition of the DF: DF of control was $21 \mathrm{D} / \mathrm{min}$, for KIC (5 mM) $10 \mathrm{D} / \mathrm{min}$, and for leucine $(5 \mathrm{mM}) 9 \mathrm{D} / \mathrm{min}$. Incubation of both $\mathrm{KIC}(5 \mathrm{mM})$ and leucine 5 $\mathrm{mM}$ resulted in a DF of $17 \mathrm{D} / \mathrm{min}$.

incubated with standard bath solution containing leucine (10 $\mathrm{mM})$ and/or KIC (10 mM).

Ligand gated channels. Neurotransmitters were applied close to the cortical neuronal soma by pulsed pressure ejection through a second micropipette (distance $30-100 \mu \mathrm{m}$ ) as described previously (7). The solution of the application pipette contained standard bath solution with the dissolved neurotransmitters in following concentrations: GABA $(100 \mu \mathrm{mol} / \mathrm{L})$ and glycine $(1 \mathrm{mM})$ or with leucine and KIC as indicated. The recordings were made in bath solution before and after incubation $(5 \mathrm{~min})$ with bath solution containing leucine $(10 \mathrm{mM})$ and/or KIC (10 mM).

Spontaneous neuronal network activity. Neuronal hippocampal and cortical cultures developed spontaneous network 


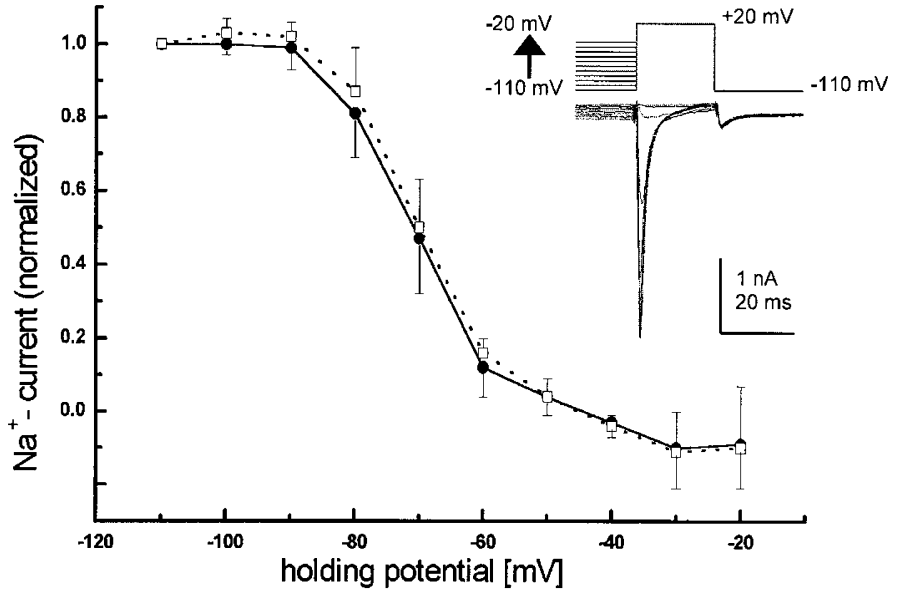

Figure 2. Leucine and $\mathrm{KIC}$ did not change the $\mathrm{Na}^{+}$-current inactivation curve. Whole-cell recording in the voltage-clamp modus in standard bath solution. Cortical neurons (DIV 10) were depolarized to $20 \mathrm{mV}$ by intracellular current application from holding potentials of $-110 \mathrm{mV}$ (see inset). The inactivation of the peak sodium current was not significantly different ( $\square$, controls; 9 , in $10 \mathrm{mM} \mathrm{KIC}$ and $10 \mathrm{mM}$ leucine; bars mean SD).

activity patterns that are based on a cooperative phenomenon of synaptic efficacy and neuronal sensitivity (8). Exposure of the neuronal cultures to $\mathrm{Mg}^{2+}$-free bath solution induced stable oscillations of membrane potential at a frequency of $\sim 0.1 \mathrm{~Hz}$. The membrane depolarizations were representing periodic bursts of action potentials that were synchronized within the cultured network (9). Increase or decrease in the burst periods indicated changes in either presynaptic or postsynaptic function. Thus, the frequency of these membrane depolarizations per minute [depolarization frequency (DF)] can be used as a value to measure the impact of neuroactive substances on the spontaneous neuronal network activity. The bath solution for induction of enhanced network activity contained (in mmol/L) $150 \mathrm{NaCl}, 0 \mathrm{MgCl}_{2}, 1 \mathrm{CaCl}_{2}, 2 \mathrm{KCl}, 10$ glucose, and 10 HEPES at $\mathrm{pH}$ 7.4. Interference by action potential generation of the recorded neuron was excluded by addition of lidocaine derivative QX-314 $(5 \mu \mathrm{M})$ to the pipette solution.

Intracellular $\mathrm{Ca}^{2+}$ measurements. Neurons were loaded with the cell permeant form of the radiometric dye Fura-2AM (5 $\mu \mathrm{M})$ for $45 \mathrm{~min}$. After dye-loading cells were perfused using the bath solution given above with leucine, KIC, or both. Glutamate was added to the perfusion solution at a final concentration of 500 $\mu \mathrm{M}$. Cells were alternately excited with light at $340 \mathrm{~nm}$ and 380 $\mathrm{nm}$ wavelength, and fluorescence was visualized using a CCD camera system (Merlin; Chromophore, Duisburg, Germany). We measured the fluorescence after excitation with light $\left(\mathrm{F}_{340}\right.$ and $\left.\mathrm{F}_{380}\right)$; calculated the ratio $\left(\mathrm{F}=\mathrm{F}_{340} / \mathrm{F}_{380}\right)$, which reflects the intracellular free $\mathrm{Ca}^{2+}$ concentration; and compared the maximal fluorescence ratio values before $\left(\mathrm{F}_{\text {before }}\right)$ and within 1 min after $\left(\mathrm{F}_{\mathrm{after}}\right)$ glutamate application. The difference of fluorescence ratios $\left(\mathrm{dF}=\mathrm{F}_{\mathrm{after}}-\mathrm{F}_{\text {before }}\right)$ is proportional to the intracellular $\mathrm{Ca}^{2+}$ concentration change (10).

Statistics. Results are given as arithmetic means \pm SEM or SD. $T$ test was used to test differences of the means at a significance level of $p<0.05$.

\section{RESULTS}

Effects on neuronal network activity. Cultured networks of hippocampal and cortical neurons [8-12 days in vitro (DIV)] developed spontaneous and stable periodic bursting as a result of long-lasting depolarizations in $\mathrm{Mg}^{2+}$-free bath solutions. After addition of $\mathrm{KIC}$ in final concentrations of 1 or $5 \mathrm{mM}$ to the bath solution, the frequency of periodic bursting decreased in all runs and spontaneous activity was even abolished at the concentration of $10 \mathrm{mM}$ (Table 1, Fig. 1A). The onset of the effect started within $10 \mathrm{~s}$ after application and reached a steady state within $1 \mathrm{~min}$ without any further decrease of the DF within the following $3 \mathrm{~min}$ recording time. The reduction of network oscillations was completely reversible after change to bath solution without KIC (Fig. $1 A)$. The same effect was observed after addition of leucine to the external bath solution (Table 1, Fig. $1 B$ ). Simultaneous incubation with both substances, leucine $(5 \mathrm{mM})$ and $\mathrm{KIC}(5 \mathrm{mM})$, which each by itself reduced DF $\sim 50 \%$, did not show the expected additive effect on DF reduction but a limited decrease to a mean of $26 \%$ (SD 14; Table 1, Fig. 1C).

Voltage-dependent currents and membrane potential. Neither action potentials (current clamp mode) nor sodium or potassium currents (voltage clamp mode) were changed in the presence of $\mathrm{KIC}$ or leucine at concentrations up to $10 \mathrm{mM}$. Especially neurons $(n=12)$ incubated for 15 min with standard bath solution containing leucine and $\mathrm{KIC}$ at a concentration of $5 \mathrm{mM}$ had maximum $\mathrm{Na}^{+}$-inward currents $\mathrm{I}_{\mathrm{Na}}=-1.44 \mathrm{nA} \pm 0.53 \mathrm{nA}$ $\left[\mathrm{I}_{\mathrm{Na}}\right.$ (control) $=-1.16 \mathrm{nA} \pm 0.46 \mathrm{nA}$, n.s. $]$ and a maximum amplitude of the voltage gated $\mathrm{K}^{+}$-outward currents $\mathrm{I}_{\mathrm{K}}=2.12 \mathrm{nA}$ $\pm 0.69 \mathrm{nA}\left[\mathrm{I}_{\mathrm{K}}\right.$ (control) $=1.85 \mathrm{nA} \pm 0.57 \mathrm{nA}$, n.s. $]$. Further detailed analysis of voltage gated $\mathrm{Na}^{+}$currents after blocking $\mathrm{K}^{+}$ currents with $\mathrm{CsCl}$ during incubation with high concentrations of leucine $(10 \mathrm{mM})$ and $\mathrm{KIC}(10 \mathrm{mM})$ did not show any significant difference of the peak values of $\mathrm{Na}^{+}$currents $\mathrm{I}_{\mathrm{Na}}=-1.62 \pm 0.8$ $\mathrm{nA}(n=9)\left[\mathrm{I}_{\mathrm{Na}}\right.$ (control) $=-1.21 \pm 0.6 \mathrm{nA}(n=6)$, n.s. $]$. Also, the corresponding $\mathrm{Na}^{+}$-channel inactivation constant $\mathrm{V} 1 / 2 \mathrm{did}$ not change during incubation $[\mathrm{V} 1 / 2=-70.2 \mathrm{mV}, \pm 2.5 \mathrm{mV}$; V1/ 2 (control) $=-69.4 \mathrm{mV}, \pm 2.1 \mathrm{mV}$, n.s.; Fig. 2]. Furthermore, the membrane potential - an integrative indicator for membrane conductances - was constant during $30 \mathrm{~min}$ of incubation with bath solution containing leucine $(10 \mathrm{mM})$ and $\mathrm{KIC}(10 \mathrm{mM})(-39.7$

Table 1. Elevated levels of leucine or KIC decreased in a dose-dependent manner the spontaneous DF of neuronal networks

\begin{tabular}{|c|c|c|c|c|c|c|}
\hline & \multicolumn{3}{|c|}{$\mathrm{KIC}$} & \multicolumn{2}{|c|}{ Leucine } & $\begin{array}{c}\text { Leucine } \\
(5 \mathrm{mmol} / \mathrm{L})+\mathrm{KIC} \\
(5 \mathrm{mmol} / \mathrm{L})\end{array}$ \\
\hline$\Delta \mathrm{DF}$ & $\begin{array}{c}28 \pm 4 \% \\
(n=4)\end{array}$ & $\begin{array}{c}50 \pm 13 \% \\
(n=3)\end{array}$ & $\begin{array}{l}\text { total abolish } \\
\quad(n=3)\end{array}$ & $\begin{array}{c}51 \pm 11 \% \\
(n=3)\end{array}$ & $\begin{array}{l}\text { total abolish } \\
\quad(n=3)\end{array}$ & $\begin{array}{c}26 \pm 14 \% \\
(n=3)\end{array}$ \\
\hline
\end{tabular}

Application of both substances resulted in a partial compensation of their effect (values given as ratio of the initial recorded frequencies from hippocampal neurons). 
$\mathrm{mV} \pm 6.15 \mathrm{mV} ; n=9)$ compared with the control group $(-35.9$ $\mathrm{mV} \pm 5.10 \mathrm{mV} ; n=6$, n.s.). Concentrations up to $10 \mathrm{mM}$ were well tolerated over hours without visible cell damage.

Postsynaptic ligand gated ion currents. Because action potential generation and voltage gated ion currents seemed to be unharmed by leucine and KIC, we investigated whether the neuronal ligand gated channels were modulated by extracellular leucine and KIC. In the first step, we filled a micropipette with $\mathrm{KIC}(10 \mathrm{mM})$ and leucine $(10 \mathrm{mM})$ and applied the solution by pressure ejection near the soma of the neurons $(n=4)$. Both substances had no effect on the neuronal membrane potential, and they did not induce any relevant membrane current (Fig. $3 A$ ). Furthermore, we did not observe any interference with the currents elicited by pressure ejection of the inhibitory neurotransmit-
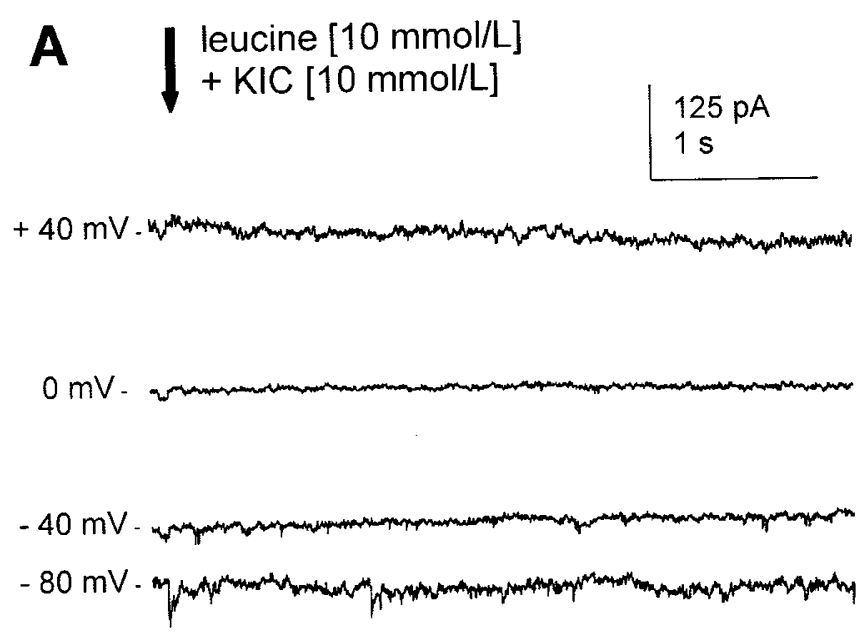

\section{B

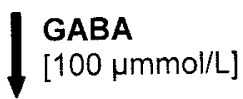

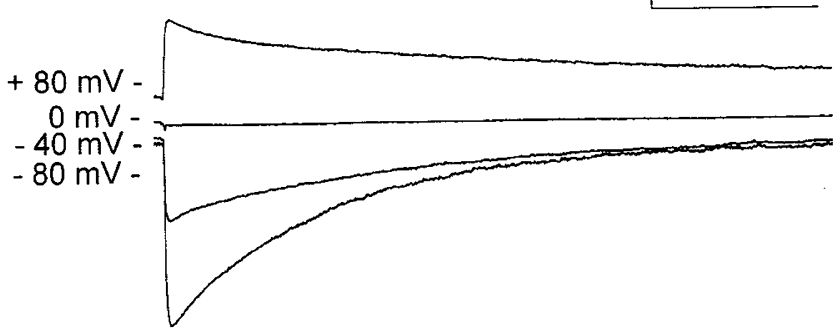

Figure 3. (a) Leucine and KIC did not possess neurotransmitter function. Whole-cell recording of a cortical neuron in the voltage-clamp modus (DIV 10 ) at various holding potentials $(40,0,-40$, and $-80 \mathrm{mV})$ in standard bath solution. Local application (10 $\mathrm{ms}$ pressure ejection) of leucine $(10 \mathrm{mM})$ and $\mathrm{KIC}(10 \mathrm{mmol} / \mathrm{L})$ near the neuronal soma did not elicit any membrane current. The vertical black arrow marks the moment of application. (B) Postsynaptic ion channels show constant membrane currents during incubation with leucine and KIC. Elicited currents after short-time application $(10 \mathrm{~ms})$ of inhibitory neurotransmitters onto the neuronal soma recorded by means of whole-cell recording in the voltage-clamp mode at various holding potentials $(-80,-40$, 0 , and $80 \mathrm{mV}$ ). The black arrow marks the moment of local GABA application after incubation with standard bath solution containing leucine $(10 \mathrm{mM})$ and KIC $(10 \mathrm{mM})$. The traces indicate normal GABA elicited chloride currents. ters GABA (100 $\mu \mathrm{mol} / \mathrm{L} ; n=3$, Fig. $3 B)$ and glycine ( $1 \mathrm{mM} ; n$ $=4$, data not shown) during incubation with bath solution containing leucine $(10 \mathrm{mM})$ and/or KIC (10 $\mathrm{mM})$.

Intracellular $\mathrm{Ca}^{2+}$ increase induced by glutamate. The neurotransmitter glutamate induces a strong intracellular calcium increase by opening of ligand gated calcium channels (10). By means of $\mathrm{Ca}^{2+}$ imaging technique, bath application of glutamate in a final concentration of $0.5 \mathrm{mM}$ induced a clear intracellular calcium response in the neuronal cells. However, there were no differences in the glutamate evoked $\mathrm{Ca}^{2+}$ responses during incubation neither with leucine $(10 \mathrm{mM})$ nor with KIC $(10 \mathrm{mM})$ alone or in combination (Table 2).

\section{DISCUSSION}

The main results of our experiments are that leucine as well as KIC reduced spontaneous neuronal network activity in a fast, reversible, and dose-dependent manner, whereas both substances antagonized each other. Leucine and KIC induced a decrease in the neuronal network DF at concentrations of 1-10 $\mathrm{mM}$, which are similar to plasma levels found in patients with MSUD during acute encephalopathic episodes $(1,5)$. The observed neurophysiological effect in the cultured network could be in principle based on alterations of the excitability of single neurons or based on changes of synaptic efficacy. In our study, impairment of the neuronal excitability seems to be unlikely because the generation of action potentials, the resting membrane potential as well as basic voltage-gated ion channels (here sodium and potassium currents), are not affected in the presence of high concentrations of leucine and/or KIC. Cultured hippocampal and cortical networks have predominantly glutamatergic and GABAergic neurons as well as glutamate, GABA, and glycine receptors $(7,11)$ driving the spontaneous network activity (12). Here, leucine and KIC failed to change the postsynaptic membrane currents elicited by glycine, GABA, and glutamate. In addition, the compounds themselves did not activate postsynaptic membrane currents, indicating that leucine and KIC have no intrinsic neurotransmitter activity.

Korein et al. (13) suggested an imbalance of presynaptic glutamate metabolism as an underlying mechanism of acute encephalopathy in patients with MSUD. Our findings also favor the hypothesis that the decrease of neuronal network activity is based on changes of presynaptic transmitter release. Glutamate is the major excitatory neurotransmitter of the CNS and the precursor of the inhibitory neurotransmitter GABA. The presynaptic glutamate synthesis requires leucine as an amino group donor that is transaminated to KIC (14). The reaction is catalyzed by the BCAA transaminase in a bidirectional enzymatic process that predicts the direction of the transamination depending on the ratio of the involved substrates: leucine $+\alpha$-ketoglutarate $\Leftrightarrow$ KIC + glutamate. Consequently, increasing concentrations of KIC results in a decrease of glutamate as described in astroglial cultures (15). In this context, a presynaptic low level of glutamate should lead to a reduced synaptic release of the excitatory neurotransmitter and thereby would explain a decrease of the observed spontaneous network activity. If this is true, we would expect a minor 
Table 2. Leucine and KIC did not impair the glutamate-induced free intracellular $\mathrm{Ca}^{2+}$ increase in cultured cortical neurons

\begin{tabular}{lcccc}
\hline & Control & Leucine $(10 \mathrm{mmol} / \mathrm{L})$ & KIC $(10 \mathrm{mmol} / \mathrm{L})$ & $\begin{array}{c}\text { KIC }(10 \mathrm{mmol} / \mathrm{L})+ \\
\text { leucine }(10 \mathrm{mmol} / \mathrm{M})\end{array}$ \\
\hline Glutamate response & $1.37 \pm 0.12$ & $1.53 \pm 0.25$ & $1.44 \pm 0.15$ & $1.47 \pm 0.29$ \\
$\mathrm{dF}$ & $(n=68)$ & $(n=91)$ & $(n=87)$ & $(n=43)$ \\
\hline
\end{tabular}

release of the transmitter into the medium. However, because of the very large "extracellular space" in monolayer cultures compared with brain tissue, we had no reliable method available for proving this hypothesis. In addition, an imbalance of spatial buffering of extracellular glutamate by glial cells seems to be unlikely because the proportion of contaminating astrocytes in our culture system is very low (6). The observation that the network activity decreases within seconds is compatible with a rapid decrease of the synaptic vesicular pool because burst of action potentials could reduce vesicular neurotransmitter release within a few seconds (16). Thus, we could not differentiate on the intracellular level which glutamate compartment - the cytosolic or the vesicular - is involved.

The surprising observation in this study was that leucine results in a similar derangement as KIC. Leucine could stimulate the activity of glutamate dehydrogenase, which may be followed by a reduction of the cytosolic or vesicular transmitter glutamate pool (17). However, an increase of leucine enhances the glutamate concentration by donating amino groups and the glutamate is metabolized to the inhibitory neurotransmitter GABA (18). Dufour et al. (19) reported neurophysiological GABAergic effects in a rat epilepsy model under increased leucine concentrations. This supports the finding that both substances functionally antagonize each other in the network activity.

The reversibility, the fast inhibition of the spontaneous network activity by leucine and KIC after a couple of seconds, and the competitive effect of KIC to leucine provide evidence for an interference of these metabolites with enzymatic stoichiometric processes or with the excitatory amino acid transport systems rather than an intoxication or even cellular destruction. With respect to microscopically unaltered morphology of cell bodies after incubation with high concentrations of leucine and KIC, we can also exclude fast damage of neuronal integrity by these substances. This is not in contradiction to the results by Jouvet et al. (4), who described a dose-dependent induction of apoptosis by KIC in cell cultures after a couple of hours. Therefore, the functional disturbance underlying acute metabolic encephalopathy has to be separated from structural CNS defects-such as dysmyelination - seen in patients with MSUD with persistently elevated leucine and KIC levels $(1,20)$.

The observed global interaction with neuronal network activity by leucine and KIC fits with clinical studies using loading tests in patients with MSUD in whom leucine and KIC were related to alterations of the EEG (13). The imbalance between concentrations of leucine and KIC could be more important for an acute encephalopathy of patients with MSUD during metabolic crisis than the absolute concentration of the single substance. Some patients showed clear neurologic signs at values of leucine $(1 \mathrm{mM})$, whereas other tolerated higher levels of leucine $(>1.5 \mathrm{mM})$ without neurologic symptoms (5).
The adaptation of patients to permanently elevated leucine/KIC concentrations could be explained by the neurophysiological antagonistic effect.

In conclusion, studies on systemic effects of metabolites on neuronal network activity seems to be helpful to elucidate pathophysiological mechanisms in acute metabolic encephalopathies.

Acknowledgments. We thank Dr. Sascha Mager for helpful discussions and Brigida Ziegler for expert technical assistance.

\section{REFERENCES}

1. Chuang DT, Shih VE 2001 Maple syrup urine disease (branched-chain ketoaciduria) In: Scriver CR, Beaudet AL, Sly WS, Valle D (eds) The Metabolic \& Molecular Bases of Inherited Disease. McGraw-Hill, New York, pp 1971-2001

2. Smith QR, Momma S, Aoyagi M, Rapoport SI 1987 Kinetics of neutral amino acid transport across the blood-brain barrier. J Neurochem 49:1651-1658

3. Zielke HR, Huang Y, Baab PJ, Collins RM, Zielke CL, Tildon JT 1997 Effect of alpha-ketoisocaproate and leucine on the in vivo oxidation of glutamate and glutamine in the rat brain. Neurochem Res 22:1159-1164

4. Jouvet P, Rustin P, Taylor DL, Pocock JM, Felderhoff-Mueser U, Mazarakis ND, Sarraf C, Joashi U, Kozma M, Greenwood K, Edwards AD, Mehmet H 2000 Branched chain amino acids induce apoptosis in neural cells without mitochondrial membrane depolarization or cytochrome c release: implications for neurological impairment associated with maple syrup urine disease. Mol Biol Cell 11:1919-1932

5. Wajner M, Coelho DM, Barschak AG, Araujo PR, Pires RF, Lulhier FL, Vargas CR 2000 Reduction of large neutral amino acid concentrations in plasma and CSF of patients with maple syrup urine disease during crises. J Inherit Metab Dis 23:505-512

6. Stichel CC, Müller HW 1992 Expression of inherent neuronal shape characteristics after transient sensitivity to epigenetic factors. Brain Res Dev Brain Res 68:149-162

7. Köller H, Siebler M, Schmalenbach C, Müller HW 1990 GABA and glutamate receptor development of cultured neurons from rat hippocampus, septal region, and neocortex. Synapse 5:59-64

8. Rose G, Siebler M 1995 Cooperative effects of neuronal ensembles. Exp Brain Res 106:106-110

9. Robinson HP, Kawahara M, Jimbo Y, Torimitsu K, Kuroda Y, Kawana A 1993 Periodic synchronized bursting and intracellular calcium transients elicited by low magnesium in cultured cortical neurons. J Neurophysiol 70:1606-1616

10. Connor JA, Tseng HY 1988 Measurement of intracellular $\mathrm{Ca} 2+$ in cerebellar Purkinje neurons in culture: resting distribution and response to glutamate. Brain Res Bull 21:353-361

11. Siebler M, Pekel M, Köller H, Müller HW 1993 Strychnine-sensitive glycine receptors in cultured primary neurons from rat neocortex. Dev Brain Res 73:289-292

12. Siebler M, Köller H, Stichel CC, Müller HW, Freund HJ 1993 Spontaneous activity and recurrent inhibition in cultured hippocampal networks. Synapse 14:206-213

13. Korein J, Sansaricq C, Kalmijn M, Honig J, Lange B 1994 Maple syrup urine disease: clinical, EEG, and plasma amino acid correlation with a theoretical mechanism of acute neurotoxicity. Int J Neurosci 79:21-45

14. Yudkoff M 1997 Brain metabolism of branched-chain amino acids. Glia 21:92-98

15. Yudkoff M, Daikhin Y, Lin ZP, Nissim I, Stern J, Pleasure D, Nissim I 2021994 Interrelationships of leucine and glutamate metabolism in cultured astrocytes. J Neurochem 62: 1192-1191

16. Naves LA, Van der Kloot W 2001 Repetitive nerve stimulation decreases the acetylcholine content of quanta at the frog neuromuscular junction. J Physiol 532:637-647

17. Plaitakis A, Zaganas I 2001 Regulation of human glutamate dehydrogenases: implications for glutamate, ammonia and energy metabolism in brain. J Neurosci Res 66:899-908

18. Waagepetersen HS, Sonnewald U, Schousboe A 1999 The GABA paradox: multiple roles as metabolite, neurotransmitter, and neurodifferentiative agent. J Neurochem 73:1335-1342

19. Dufour F, Nalecz KA, Nalecz MJ, Nehlig A 2001 Modulation of absence seizures by branched-amino acids: correlation with brain amino concentrations. Neurosci Res 40:255-263

20. Treacy E, Clow CL, Reade TR, Chitayat D, Manner OA, Scriver CR 1992 Maple syrup urine disease: interrelations between branched-chain amino-, oxo- and hydroxyacids; implications for treatment; association with dysmyelination. J Inherit Metab Dis 15:121-126 